\title{
Exercise is associated with improved asthma control in adults
}

\author{
S. Dogra*,\# , J.L. Kuk ${ }^{\#}$, J. Baker*,\# and V. Jamnik ${ }^{\#, \oplus}$
}

\section{ABSTRACT: Global asthma control levels are suboptimal. The influence of regular exercise on asthma control is unclear.}

We assessed the effects of a 12-week supervised exercise intervention followed by 12 weeks of self-administered exercise on adults with partially controlled asthma $(n=21)$ and matched controls $(n=15)$. Assessments were conducted at baseline and week 12 for both the exercise and control group, and again at week 24 for the exercise group.

There was a significant treatment effect on asthma control in the exercise group, as measured by the Asthma Control Questionnaire (ACQ), from baseline to week 12, compared with the control group. A clinically significant improvement ( 0.5 increase in ACQ score) was observed for asthma quality of life and $A C Q$ in the exercise group from baseline to week 12. There was a significant improvement in aerobic fitness from baseline to week 24 in the exercise group.

In conclusion, a 12-week supervised exercise intervention led to improvements in asthma control and quality of life in partially controlled asthmatics motivated to exercise. These improvements were maintained, while aerobic fitness and perceived asthma control significantly improved over an additional 12 weeks of self-administered exercise. These findings indicate that a structured exercise intervention can improve asthma control.

KEYWORDS: Aerobic fitness, asthma, exercise, perceived asthma control, quality of life

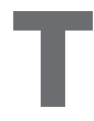
here is no known cure for asthma [1, 2]; however, pharmacological intervention has been shown to significantly improve symptoms [3-5]. The use of inhaled corticosteroids, long acting $\beta_{2}$-agonists and a combination of these medications have been shown to improve asthma control $[3,4]$, but compliance rates of $>80 \%$ are required to maintain this level of control [6]. Unfortunately, compliance to asthma treatment in countries where treatment is readily accessible remains poor $[7,8]$.

Asthma control is determined by the frequency of daytime symptoms, limitation of activities, nocturnal symptoms, need for reliever medication, lung function and exacerbations [1]. Accordingly, patients are classified as having controlled, partially controlled or uncontrolled asthma. Recent data show that only $23 \%$ of asthmatics are controlled [9] and, despite receiving specialist care, 50\% are not well controlled [8]. Poor asthma control has been associated with more emergency room visits, physician visits and days spent in hospital [10].

Recent research demonstrates that healthcare use is higher in physically inactive asthmatics compared with physically active asthmatics [11]. This finding suggests that active asthmatics have better asthma control, if healthcare use is a proxy of asthma control. Exercise interventions involving adults with asthma have shown improvements in measures such as lung function [12], quality of life [13], breathlessness [14, 15] and controller therapy [16], while animal models have shown improvements in airway inflammation $[17,18]$. However, a direct association between asthma control and exercise has not yet been made.

VOLLMER et al. [19] reported that the activity limitation component, which includes both physical and nonphysical activity, is the most powerful contributor to asthma control, suggesting that improvements in aerobic fitness may improve control in partially controlled but physically inactive asthmatics. To date, the benefits of exercise interventions have been demonstrated by medically supervised programmes $[16,20]$. The effects of such programmes on future physical activity habits are unclear; furthermore, the effects of self-administered exercise programmes remain unknown. Self-administered exercise may be a more cost-effective and readily available therapy for the general population of adults with asthma. If exercise positively impacts asthma control, it may be an important adjunct
AFFILIATIONS

*Lifespan, Health and Performance Laboratory,

"Human Performance Laboratory, and

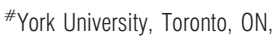

Canada.

CORRESPONDENCE

S. Dogra

School of Recreation Management and Kinesiology

Acadia University

550 Main St

Wolfville NS

B4P-2R6

Canada

E-mail: shilpa.dogra@acadiau.ca

Received:

Nov 162009

Accepted after revision:

May 282010

First published online:

June 072010

European Respiratory Journa

Print ISSN 0903-1936

Online ISSN 1399-3003 
therapy for adults with partially controlled asthma and poor compliance to prescribed medication.

It is, therefore, imperative to gain a better understanding of the effect of regular exercise on asthma control and determine whether there is a clinically relevant benefit. The purpose of the current investigation was to determine 1) whether a 12week supervised exercise programme leads to improvements in asthma control and aerobic fitness levels in partially controlled adults with asthma compared to a matched control group and 2) whether the exercise group would be able to maintain the benefits from the programme after an additional 12 weeks of self-administered exercise.

\section{METHODS}

\section{Participants}

Participants were recruited from the Greater Toronto Area in Ontario, Canada. Inclusion was limited to male and female adults over the age of 18 yrs, who were not pregnant, had a current prescription for asthma medication and were physically inactive as defined by the Canadian Physical Activity Guidelines [21]. All participants provided informed consent prior to testing and the study protocol was approved by the Human Participants Review Sub-Committee of the Ethics Committee of York University (Toronto, ON, Canada).

\section{Study design}

The study was a nonrandomised control trial. Placement in the exercise group was based on availability to meet with a qualified exercise professional (Canadian Society for Exercise Physiology Certified Exercise Physiologist $\mathbb{R}$; CSEP-CEP) three times per week for 12 weeks and the ability to exercise uninterrupted for the study duration. All participants in the exercise group were recruited in the autumn season and completed the 24-week programme by March. The control group was matched for age and sex, and was provided with an exercise programme upon completing the control period. The control group was recruited on a rolling intake from autumn to spring.

\section{Exercise programme}

Participants in the exercise group were assigned to a CSEPCEP with whom they exercised three times per week for 12 weeks. This was followed by an additional 12-week period of self-administered exercise, as prescribed by the CSEP-CEP. The self-administered programme was individualised, based on the participant's access to equipment, physical activity preferences, and short- and long-term fitness goals. The control group was directed to maintain their current lifestyle.

The main focus of the exercise sessions was aerobic training, but one set per week of strength exercises targeting the major muscle groups (chest, back, shoulders, triceps, biceps, quadriceps, hamstrings and abdominals) was also included. The mode of aerobic exercise was based on individual preference, but was limited to outdoor jogging, treadmill, recumbent or upright cycling, and elliptical or rowing machines. The intensity for aerobic exercise was based on maximum heart rate (HRmax) obtained during the aerobic fitness test. Since participants were previously sedentary, the programme intensity increased progressively by $5 \%$ every 3 weeks from a minimum of $70 \%$ HRmax to a minimum of $85 \%$ HRmax. Participants were required to wear heart rate (HR) monitors
(Polar S625x; Polar Electro, Kempele, Finland) during these exercise sessions, to ensure that the minimum HR was achieved. They were also shown the Borg Rating of Perceived Exertion [22] scale at each 5-min interval of exercise so that the participants understood the "feeling" associated with that intensity of exercise; this was particularly important for the self-administered exercise portion of the programme. Participants were permitted to use their bronchodilators before or during exercise sessions if necessary.

Self-administered exercise programmes provided to the exercise group for the second 12-week period included aerobic exercise at $85 \%$ HRmax and resistance exercises. Participants were provided with logs, which were submitted at the follow-up testing session. They were encouraged to exercise 5 days $\cdot$ week $^{-1}$.

\section{Testing sessions}

Participants were given standard instructions to follow before coming to the laboratory: 1) no short-acting bronchodilators in the $6 \mathrm{~h}$ prior to the testing session; 2) no caffeine in the $6 \mathrm{~h}$ prior to the testing session; 3) no alcohol or heavy exercise in the $24 \mathrm{~h}$ prior to the testing session; and 4) no smoking in the $8 \mathrm{~h}$ prior to the testing session. Participants were not instructed to discontinue use of controller medication, as the lung function tests and the exercise test were not intended to be diagnostic tools.

\section{Spirometry}

Forced expiratory volume in $1 \mathrm{~s}$ (FEV1) and forced vital capacity (FVC) were measured using a handheld spirometer (MicroSpirometer; Vacumed, Ventura, CA, USA). The prebronchodilator FEV1 was used to determine FEV1 \% predicted (\% pred), which was required for the Asthma Control Questionnaire (ACQ) [23]. Participants were asked to take their short-acting bronchodilator to ensure that the exercise test would not be terminated due to exercise-induced asthma symptoms. FEV1 and FVC were measured 15 min after use of the bronchodilator and then again $10 \mathrm{~min}$ following the aerobic fitness test.

\section{Aerobic fitness}

Participants completed an incremental aerobic fitness test (maximal oxygen uptake $\left.\left(V^{\prime} \mathrm{O}_{2}, \max \right)\right)$ on a treadmill. The associated measurements (fractional concentrations of oxygen and carbon dioxide, and minute ventilation) were determined directly via the open-circuit technique with discrete components (120-L Tissot spirometer, and applied electrochemistry oxygen and carbon dioxide analysers). The loading protocol varied depending on the participant's comfort level and ability to run. Participants started with a 3-min walking workload and progressed from walking (4.83-6.44 $\left.\mathrm{km} \cdot \mathrm{h}^{-1} ; 3.0-4.0 \mathrm{mph}\right)$ to jogging $\left(8.05-9.66 \mathrm{~km} \cdot \mathrm{h}^{-1} ; 5.0\right.$ $6.0 \mathrm{mph})$ to running $\left(11.27-12.87 \mathrm{~km} \cdot \mathrm{h}^{-1} ; 7.0-8.0 \mathrm{mph}\right)$; subsequent work rates were increased by increasing the incline by $2 \%$ per workload. Participants who were unable to run completed a walking protocol that progressed from walking on a low $(0-5 \%)$ gradient, which increased by $2 \%$ every $2 \mathrm{~min}$. The attainment of $V^{\prime} \mathrm{O}_{2}$,max was confirmed when oxygen uptake $\left(V^{\prime} \mathrm{O}_{2}\right)$ plateaued or decreased with progressively increasing work rates. In most instances, the criteria for $V^{\prime} \mathrm{O}_{2}$ max was volitional fatigue and, therefore, the aerobic fitness test did not provide a true $V^{\prime} \mathrm{O}_{2}$, max but, rather, a peak $V^{\prime} \mathrm{O}_{2}\left(V^{\prime} \mathrm{O}_{2}\right.$,peak). Peak minute ventilation $\left(V^{\prime} \mathrm{E}\right)$ 


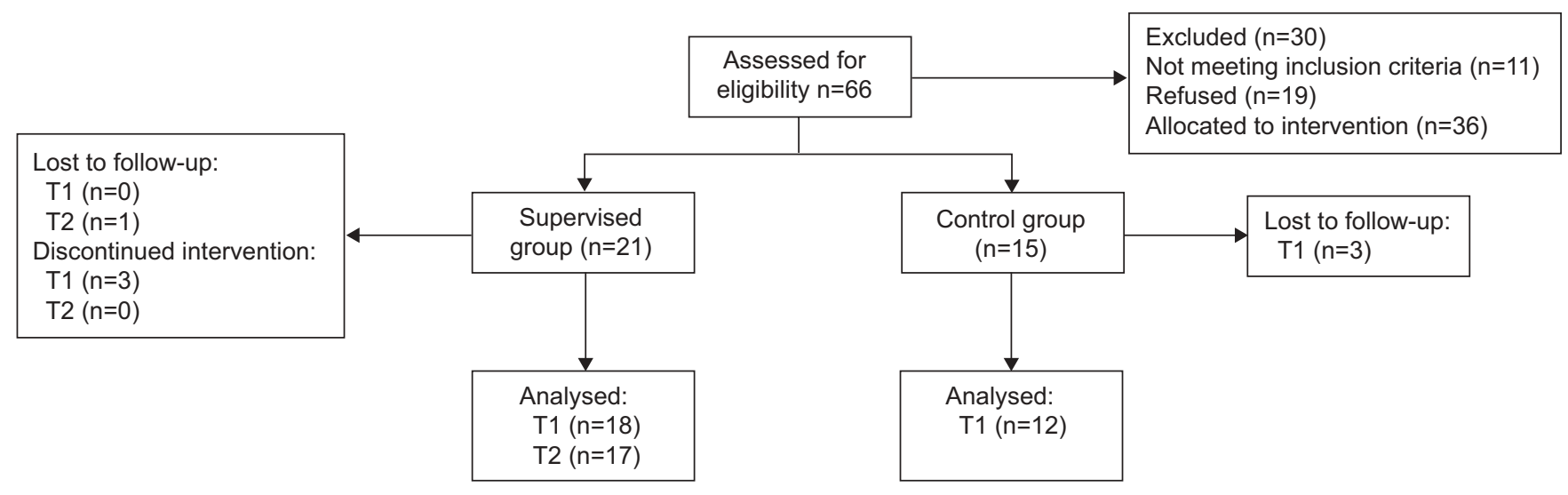

FIGURE 1. Flow chart for recruitment and intervention period. T1: baseline; T2: week 12.

was used to calculate ventilatory reserve or dyspnoea index (DI; $\left.V^{\prime} \mathrm{E} /(\mathrm{FEV} 1 \times 35)\right)$ and ventilatory efficiency (ventilatory equivalent for oxygen $\left.\left(V^{\prime} \mathrm{E} / V^{\prime} \mathrm{O}_{2}\right)\right)$ at $75 \% V^{\prime} \mathrm{O}_{2}$,peak (submaximal) and $V^{\prime} \mathrm{O}_{2}$,peak for each participant. DI has been used in research related to chronic obstructive pulmonary conditions as it is a reliable indicator of ventilatory reserve [20, 24].

\section{Questionnaires}

The main outcome measure, asthma control, was measured using the ACQ [23] and a single-item perceived asthma control question. The ACQ is a validated questionnaire wherein a score of $<0.75$ indicates well-controlled asthma, $0.75-1.5$ denotes relatively well-controlled asthma and $>1.5$ indicates poorly controlled asthma. This questionnaire can be used with or without spirometric measures [23]. The single-item perceived asthma control variable was a multiple choice question with the following options for current control levels: 1) total control 2) well controlled 3) moderately controlled 4) low control and 5) poor control. This variable was included in the study as perceived control is significantly associated with activity restriction in adults with asthma [25]. Quality of life was assessed using the mini-Asthma Quality of Life Questionnaire (mini-AQLQ) [26], wherein higher scores are indicative of better quality of life. Additional information pertaining to demographics, asthma symptoms and physical activity history was also collected.

\section{Statistical analyses}

Baseline characteristics between groups were compared using independent sample unpaired t-tests and Chi-squared tests. ANCOVAs were used to determine treatment differences between groups over the 12-week intervention, adjusting for baseline values. Repeated-measures ANOVAs were conducted to determine treatment effects over the 24-week period from baseline and week 12 in the exercise group. Power calculations were conducted for the primary outcome. All statistical analyses were conducted using SPSS 17.0 (SPSS Inc., Chicago, IL, USA).

\section{RESULTS}

A total of 66 participants were assessed for eligibility (fig. 1). 11 participants were ineligible, as they were physically active, while 19 declined to participate in the study. 21 participants were placed in the exercise and 15 in the control group.
Accounting for dropouts and loss to follow-up, a total of 18 participants in the exercise and 12 in the control group were included in the analyses.

There were no differences in the baseline characteristics of the participants between groups (table 1). Lung function measurements, either before or after the use of a bronchodilator, or after exercise, were similar between groups at baseline (table 2). There were no changes in lung function measurements expressed in terms of FEV1/FVC or FEV1 \% pred after the intervention period or at the follow-up period for the exercise group.

The groups differed significantly at baseline for ACQ with spirometry $(p<0.05)$. Over the supervised treatment period, ACQ without spirometry significantly improved in the exercise group compared with the control group (table 3). However, ACQ score with spirometry did not improve significantly in the exercise group compared with the control group. There was a trend for significant improvements in perceived asthma control $(\mathrm{p}=0.051)$, submaximal $V^{\prime} \mathrm{E} / V^{\prime} \mathrm{O}_{2}$

\begin{tabular}{|c|c|c|}
\hline \multirow[t]{2}{*}{ TABLE 1} & \multirow[b]{2}{*}{ Control group } & \multirow[b]{2}{*}{ Exercise group } \\
\hline & & \\
\hline Subjects & 12 & 18 \\
\hline Age yrs & $34.0 \pm 3.4$ & $34.2 \pm 3.2$ \\
\hline Females & 9 & 13 \\
\hline BMI $\mathbf{k g} \cdot \mathrm{m}^{-2}$ & $24.4 \pm 1.1$ & $25.8 \pm 0.9$ \\
\hline $\begin{array}{l}\text { Prescribed preventive } \\
\text { medicine }\end{array}$ & 9 & 11 \\
\hline $\begin{array}{l}\text { Prescribed rescue } \\
\text { medicine }\end{array}$ & 12 & 16 \\
\hline Presence of EIA & 11 & 14 \\
\hline Presence of allergies & 11 & 17 \\
\hline Never-smokers & 4 & 12 \\
\hline Pre-BD FEV1 & $2.86 \pm 0.18$ & $2.96 \pm 0.26$ \\
\hline
\end{tabular}


TABLE 2 Lung function parameters by group for each time-point of the intervention

\begin{tabular}{|c|c|c|c|c|c|}
\hline & Baseline & Week 12 & Baseline & Week 12 & Week 24 \\
\hline Subjects $n$ & 12 & 11 & 18 & 18 & 16 \\
\hline Pre-BD FEV $1 /$ FVC $\%$ & $82.6 \pm 2.2$ & $80.2 \pm 2.1$ & $80.2 \pm 2.1$ & $78.5 \pm 2.26$ & $78.4 \pm 2.8$ \\
\hline Post-BD FEV $1 /$ FVC $\%$ & $84.1 \pm 2.5$ & $83.2 \pm 2.1$ & $81.8 \pm 2.0$ & $82.8 \pm 2.0$ & $81.0 \pm 2.6$ \\
\hline Post-BD FEV 1 \% pred & $90.5 \pm 4.9$ & $92.1 \pm 4.6$ & $88.9 \pm 3.7$ & $88.9 \pm 2.9$ & $83.6 \pm 4.1$ \\
\hline Post-exercise FEV $1 \%$ pred & $87.8 \pm 4.7$ & $87.2 \pm 4.2$ & $87.3 \pm 3.6$ & $84.2 \pm 3.6$ & $80.0 \pm 4.7$ \\
\hline
\end{tabular}

Data are presented as mean \pm SE unless otherwise stated. BD: bronchodilator; FEV1: forced expiratory volume in 1 s; FVC: forced vital capacity; \% pred: \% predicted.

$(\mathrm{p}=0.078)$ and submaximal DI $(\mathrm{p}=0.054)$ in the exercise group. With self-administered exercise, there was a significant improvement in mini-AQLQ and a trend toward significance $(\mathrm{p}=0.073)$ for $V^{\prime} \mathrm{E} / V^{\prime} \mathrm{O}_{2}$ at peak from week 12 to week 24 . Furthermore, there were significant improvements in ACQ without spirometry, mini-AQLQ, perceived asthma control, $V^{\prime} \mathrm{O}_{2}$, peak and submaximal DI from baseline to week 24 in the exercise group. A clinically significant improvement of $>0.5$ was observed in both the ACQ and mini-AQLQ from baseline to week 12 , and was maintained over the follow-up period to week 24.

\section{Adverse events}

One participant experienced an acute attack of asthma that required hospitalisation during the self-administered exercise period; this did not occur during an exercise session. The attack was thought to be the result of illness that stemmed from a visit abroad and not due to the exercise programme. This participant willingly resumed exercise and attended the follow-up fitness test at week 24 . One participant injured their ankle during the self-administered exercise period. Although we can not say with certainty that this was not a result of the prescribed exercise, the participant claimed to have previous problems with this ankle that became aggravated. No other adverse events were reported. There were no incidents related to exercise-induced asthma over the course of the intervention.

\section{DISCUSSION}

The effect of a 12-week supervised exercise intervention on asthma control was assessed in adults with partially controlled asthma who were interested in beginning an exercise programme. Our primary finding is that a 12-week supervised exercise intervention leads to statistically and clinically significant improvements in asthma control compared with a matched control group. This may be attributed to improvements in submaximal measures (at $75 \%$ ) of aerobic fitness, such as $V^{\prime} \mathrm{E} / V^{\prime} \mathrm{O}_{2}$ and DI. Furthermore, supervised exercise followed by a period of self-administered exercise leads to improvements in $V^{\prime} \mathrm{O}_{2}$, peak, quality of life and maintenance of asthma control.

\section{TABLE 3 Main outcome variables at baseline and follow-up}

\begin{tabular}{|c|c|c|c|c|c|}
\hline & \multicolumn{2}{|c|}{ Control group } & \multicolumn{3}{|c|}{ Exercise group } \\
\hline & Baseline & Week 12 & Baseline & Week 12 & Week 24 \\
\hline Subjects n & 12 & $11^{\bullet}$ & 18 & 18 & $15^{f}$ \\
\hline$A C Q$ & $0.90 \pm 0.15$ & $0.99 \pm 0.16^{\bullet}$ & $1.30 \pm 0.19$ & $0.72 \pm 0.10^{+}$ & $0.72 \pm 0.17^{f, \# \#}$ \\
\hline$A C Q$ with spirometry & $1.06 \pm 0.10^{\#}$ & $0.80 \pm 0.14$ & $1.37 \pm 0.21$ & $0.95 \pm 0.11$ & $1.02 \pm 0.18$ \\
\hline$V^{\prime} \mathrm{O}_{2}, \max$ & $2.66 \pm 0.27$ & $2.77 \pm 0.29$ & $2.63 \pm 0.20$ & $2.88 \pm 0.21$ & $3.00 \pm 0.27^{\# \#}$ \\
\hline Submaximal $V^{\prime} E / V^{\prime} O_{2}$ & $23.21 \pm 0.73$ & $23.64 \pm 0.80$ & $24.89 \pm 1.08$ & $23.78 \pm 0.74^{\S}$ & $24.35 \pm 0.90$ \\
\hline Maximal $V^{\prime} E / V^{\prime} O_{2}$ & $28.46 \pm 0.88$ & $28.65 \pm 1.39$ & $28.84 \pm 1.02$ & $27.44 \pm 0.78$ & $28.48 \pm 1.17$ \\
\hline Submaximal DI & $0.42 \pm 0.03$ & $0.42 \pm 0.02$ & $0.45 \pm 0.02$ & $0.48 \pm 0.03^{\S}$ & $0.54 \pm 0.03^{\# \#}$ \\
\hline Maximal DI & $0.68 \pm 0.04$ & $0.69 \pm 0.04$ & $0.73 \pm 0.04$ & $0.77 \pm 0.04$ & $0.84 \pm 0.04$ \\
\hline
\end{tabular}

Data are presented as mean \pm SE unless otherwise stated. ACQ: Asthma Control Questionnaire; mini-AQLQ: mini-Asthma Quality of Life Questionnaire; $V^{\prime} \mathrm{O}_{2}$,max: maximal oxygen uptake; $V^{\prime} \mathrm{E}$ : minute ventilation; $\mathrm{V}^{\prime} \mathrm{O}_{2}$ : oxygen uptake; $\mathrm{DI}$ : dyspnoea index. ${ }^{*}$ : significant differences at baseline between control and exercise group; ${ }^{\circ}: \mathrm{n}=12$ for paper measurements only; ${ }^{+}: p<0.05$ between groups from baseline to week $12 ;{ }^{\S}: p<0.10$ statistical trend from baseline to week $12 ;{ }^{f}: n=17$ for paper measurements only; ${ }^{\# \#: ~} p<0.05$ within exercise group from weeks 12 to $24 ; "$ " $p<0.05$ within exercise group from weeks 12 to 24 . 
Our finding that quality of life improved with supervised exercise is consistent with previous research $[12,13]$. However, the improvement in asthma control with supervised exercise is a novel finding and highlights the importance of encouraging exercise in this population. While previous research has concluded that exercise leads to improvements in medication use [13], the frequency of exercise-induced asthma [27] and lung function [12], this study is the first to show that regular exercise participation can lead to a change in overall asthma control. It is important to highlight that there were also clinically relevant improvements in mean ACQ score and mini-AQLQ score from baseline to week 12 in the exercise group.

Only one previous study has attempted to assess the association between exercise and asthma control. FANELLI et al. [13] conducted a 16-week exercise program in children with asthma, for which the children exercised for 90 min twice per week. They showed significant improvements in aerobic fitness, improvements in asthma quality of life and reduction in medication use. Although medication use is a component of asthma control, the authors did not measure overall asthma control. Our analysis fuses a validated asthma control questionnaire (ACQ) and perceived asthma control. Previous research has shown that greater perceived control is associated with improved measures of physical and mental health status, better quality of life and, most importantly, fewer days of restricted activity (physical and nonphysical) due to asthma [25]. That we observed improvements in both measured and perceived asthma control strengthen our findings and reinforce the importance of regular exercise participation for adults with asthma.

The self-administered exercise was a unique aspect of this study. Participants in the exercise group were provided with an exercise programme to execute on their own after week 12. This resulted in significant improvements in quality of life and maintenance of asthma control, suggesting that, after an initial supervised exercise period, adults with partially controlled asthma can exercise with continued success. These findings are similar to those reported by EMTNER et al. [12], where a 10-week exercise programme in well-controlled asthmatic adults led to improvements in exercise capacity, lung function and asthma severity. Interestingly, $68 \%$ of the adults with asthma from that study were still exercising at least once per week 3 yrs later, and also had decreased medication use and emergency room visits, both of which are proxy measures of asthma control [28].

It is possible that a greater frequency, duration or intensity of the exercise intervention was required to induce significant changes in measures of aerobic fitness; however, we believe that it was important to use an exercise intervention that is similar to the guidelines recommended by public health agencies $[21,29]$. These physical activity guidelines are readily accessible to the public and are easy to interpret by physicians and their patients. It is apparent that the use of these minimum physical activity recommendations as an adjunct therapy for asthma could relieve some of the healthcare burden associated with asthma [30,31] and counter the impact of poor adherence to medication [32]. Lack of significant improvements in measures of aerobic fitness may also be related to power, as a sample size of 20 subjects per group $(n=18$ in the present study) would be required for $80 \%$ power at a $\mathrm{p}<0.05$, based on the variability within the exercise group [33].

It should be emphasised that all participants in the exercise group became physically active, and only two participants in the exercise group did not attend $100 \%$ of the sessions, but attended over $70 \%$ of the sessions. This small increase in exercise level led to a significant improvement in perceived and measured asthma control. These results are consistent with the finding by VOLLMER et al. [19] indicating that activity restriction (physical and nonphysical) is a powerful predictor of asthma control. This is important, as poor asthma control is associated with greater healthcare use, and supervised exercise followed with self-administered exercise may be a sustainable solution to improve asthma control and relieve some of the burden on public healthcare systems.

The results of this study should be interpreted in light of the following limitations. 1) We demonstrate changes in ACQ in the exercise group, but the current analysis was underpowered for seeing treatment effects using ACQ with spirometry. Although the use of ACQ without spirometry is a valid method [23] based on previous studies [14], resting spirometric values are not expected to change with regular exercise. Therefore, use of the ACQ without spirometry is perhaps an equivalent measure for longitudinal changes with exercise. 2) We did not have any clinical measures of asthma control or severity, such as peak flow variability or exhaled nitric oxide. 3) We did not have objective physical activity monitors and are, therefore, unable to say what level of physical activity and exercise occurred outside the supervised sessions. 4) We were unable to randomise the sample into intervention groups due to logistics pertaining to gym access and time. 5) The season in which the groups were recruited varied somewhat and may have led to the slight improvements observed in the control group; however, despite this, the exercise group had a significant improvement in ACQ compared to control.

In conclusion, we found that a 12-week supervised exercise intervention led to improvements in asthma control and quality of life in partially controlled adults with asthma who were interested in exercising. Additionally, supervised exercise followed by a period of self-administered exercise maintained the improved asthma control levels and resulted in significant improvements in aerobic fitness and perceived asthma control. These findings indicate that a structured exercise intervention can improve asthma control. Future research should determine the feasibility of including exercise programs as an essential adjunct to asthma management programs.

\section{SUPPORT STATEMENT}

This work was supported in part by a York University Faculty of Health Minor Research Award, and a Social Sciences and Humanities Research Council of Canada Small Grant.

\section{STATEMENT OF INTEREST}

None declared.

\section{ACKNOWLEDGEMENTS}

The authors would like to thank all of the CSEP-CEPs that assisted with this research. 


\section{REFERENCES}

1 Global Initiative for Asthma. Global Strategy for Asthma Management and Prevention. GINA. www.ginasthma.org. Date last accessed: August 12, 2009. Date last updated: 2009.

2 Warner JO, Naspitz CK. Third international pediatric consensus statement on the management of childhood asthma. Pediatr Pulmonol 1998; 25: 1-17.

3 O'Byrne PM, Naya IP, Kallen A, et al. Increasing doses of inhaled corticosteroids compared to adding long-acting inhaled $\beta_{2-}$ agonists in achieving asthma control. Chest 2008; 134: 1192-1199.

4 Lalloo UG, Malolepszy J, Kozma D, et al. Budesonide and formoterol in a single inhaler improves asthma control compared with increasing the dose of corticosteroid in adults with mild-tomoderate asthma. Chest 2003; 123: 1480-1487.

5 Foresi A, Morelli MC, Catena E. Low-dose budesonide with the addition of an increased dose during exacerbations is effective in long-term asthma control. On behalf of the Italian study group. Chest 2000; 117: 440-446.

6 Lasmar L, Camargos P, Champs NS, et al. Adherence rate to inhaled corticosteroids and their impact on asthma control. Allergy 2009; 64: 784-789.

7 Legorreta AP, Christian-Herman J, O'Connor RD, et al. Compliance with national asthma management guidelines and specialty care: a health maintenance organization experience. Arch Intern Med 1998; 158: 457-464.

8 Gaga M, Papageorgiou N, Zervas E, et al. Control of asthma under specialist care: is it achieved? Chest 2005; 128: 78-84.

9 Chapman KR, Boulet LP, Rea RM, et al. Suboptimal asthma control: prevalence, detection and consequences in general practice. Eur Respir J 2008; 31: 320-325.

10 Williams SA, Wagner S, Kannan $\mathrm{H}$, et al. The association between asthma control and health care utilization, work productivity loss and health-related quality of life. J Occup Environ Med 2009; 51: 780-785.

11 Dogra S, Baker J, Ardern CI. The role of physical activity and body mass index in the health care use of adults with asthma. Ann Allergy Asthma Immunol 2009; 102: 462-468.

12 Emtner M, Herala M, Stålenheim G. High-intensity physical training in adults with asthma. A 10-week rehabilitation program. Chest 1996; 109: 323-330.

13 Fanelli A, Cabral AL, Neder JA, et al. Exercise training on disease control and quality of life in asthmatic children. Med Sci Sports Exerc 2007; 39: 1474-1480.

14 Cochrane LM, Clark CJ. Benefits and problems of a physical training programme for asthmatic patients. Thorax 1990; 45: 345-351.

15 Clark CJ. The role of physical training in asthma. Chest 1992; 101 Suppl. 5, 293S-298S.

16 Neder JA, Nery LE, Silva AC, et al. Short-term effects of aerobic training in the clinical management of moderate to severe asthma in children. Thorax 1999; 54: 202-206.
17 Pastva A, Estell K, Schoeb TR, et al. Aerobic exercise attenuates airway inflammatory responses in a mouse model of atopic asthma. J Immunol 2004; 172: 4520-4526.

18 Vieira RP, Claudino RC, Duarte AC, et al. Aerobic exercise decreases chronic allergic lung inflammation and airway remodeling in mice. Am J Respir Crit Care Med 2007; 176: 871-877.

19 Vollmer WM, Markson LE, O'Connor E, et al. Association of asthma control with health care utilization: a prospective evaluation. Am J Respir Crit Care Med 2002; 165: 195-199.

20 Hallstrand TS, Bates PW, Schoene RB. Aerobic conditioning in mild asthma decreases the hyperpnea of exercise and improves exercise and ventilatory capacity. Chest 2000; 118: 1460-1469.

21 Public Health Agency of Canada. Canada's Physical Activity Guide to Healthy Active Living. www.phac-aspc.gc.ca/pau-uap/ paguide/ Date last accessed: August 12, 2009. Date last updated: September 3, 2010.

22 Borg GA. Psychophysical bases of perceived exertion. Med Sci Sports Exerc 1982; 14: 377-381.

23 Juniper EF, O'Byrne PM, Guyatt GH, et al. Development and validation of a questionnaire to measure asthma control. Eur Respir J 1999; 14: 902-907.

24 Fink G, Moshe S, Goshen J, et al. Functional evaluation in patients with chronic obstructive pulmonary disease: pulmonary function test versus cardiopulmonary exercise test. J Occup Environ Med 2002; 44: 54-58.

25 Calfee CS, Katz PP, Yelin EH, et al. The influence of perceived control of asthma on health outcomes. Chest 2006; 130: 1312-1318.

26 Juniper EF, Guyatt GH, Cox FM, et al. Development and validation of the mini asthma quality of life questionnaire. Eur Respir J 1999; 14: 32-38.

27 Bonsignore MR, La Grutta S, Cibella F, et al. Effects of exercise training and montelukast in children with mild asthma. Med Sci Sports Exerc 2008; 40: 405-412.

28 Emtner M, Finne M, Stålenheim G. A 3-year follow-up of asthmatic patients participating in a 10-week rehabilitation program with emphasis on physical training. Arch Phys Med Rehabil 1998; 79: 539-544.

29 Haskell WL, Lee IM, Pate RR, et al. Physical activity and public health: updated recommendation for adults from the American College of Sports Medicine and the American Heart Association. Med Sci Sports Exerc 2007; 39: 1423-1434.

30 Bahadori K, Doyle-Waters MM, Marra C, et al. Economic burden of asthma: a systematic review. BMC Pulm Med 2009; 9: 24.

31 Cisternas MG, Blanc PD, Yen IH, et al. A comprehensive study of the direct and indirect costs of adult asthma. J Allergy Clin Immunol 2003; 111: 1212-1218.

32 Maneechotesuwan K, Essilfie-Quaye S, Kharitonov SA, et al. Loss of control of asthma following inhaled corticosteroid withdrawal is associated with increased sputum interleukin-8 and neutrophils. Chest 2007; 132: 98-105.

33 Overall JE, Doyle SR. Estimating sample sizes for repeated measurement designs. Controlled Clin Trials 1994; 15: 100-123. 